\title{
Calculation of Equivalent Square Fields in Radiation Therapy by an Improved Vadash Correction Factor for Collimator Exchange Effects
}

\author{
Mohammad Javad Tahmasebi Birgani ${ }^{1}$, Nahid Chegeni' ${ }^{2}$, Marziyeh Tahmasbi ${ }^{3}$, \\ Raziye Fayazi ${ }^{2 *}$ Jafar Fatahi Asl ${ }^{3}$, Mohmmad Ali Behrouz ${ }^{2}$
}

\begin{abstract}
Background and objectives: To determine the head scatter factor, a formalism presented by Vadash and Bjärngard has been employed to assess collimator exchange effects. The aim of this study was to determine the best Vadash correction factor (A) by introducing a new method based on the output factor measured in air for different square and rectangular fields. Materials and Methods: A new simple mathematical method based on selection of the best dosimetric data was proposed to obtain the A value for Vadash to predict the equivalent square field size. Measurements were performed with a Farmer chamber $0.6 \mathrm{cc}$ in SSD $100 \mathrm{~cm}$ and build up cap of Plexiglas, 1.5 and $3.5 \mathrm{~cm}$ equal to water, for 6 and $18 \mathrm{MV}$, respectively, with a Perimus Plus linear accelerator. The output factor in air $\left(\mathrm{OF}_{\text {air }}\right)$ was measured for square and rectangular fields. MATLAB software (version R2014a) was employed for calculations and curve fitting. Results: A power model with a constant value was applied to the output factor in air as a function of square field size. The output factor in air ranged from 0.983 to 1.038 at $6 \mathrm{MV}$ and from 0.731 to 1.05 at $18 \mathrm{MV}$ with the Y collimator having a greater effect. Obtained values for A were 1.42 and 1.55 with respect to $\sigma_{\min }=0.98$ and 2.3 for 6 and $18 \mathrm{MV}$ energies, respectively. Conclusion: The proposed A values minimize the collimator exchange effect in calculating equivalent squares, which plays an important role in patient dose calculation and treatment planning.
\end{abstract}

Keywords: Equal square field- collimator exchange effect- Vadash formula- Output factors in air

Asian Pac J Cancer Prev, 19 (11), 3053-3057

\section{Introduction}

Radiation therapy is currently one of the most important therapeutic methods for cancer treatment. The aim of radiation therapy is to maximize damage to the tumor while protecting of the normal tissues (Shalek, 1977; Van Herk, 2004). An important issue in radiotherapy is dose calculating and treatment planning for cancer patients. Photon beam data, such as percentage depth doses, dose profiles, and output factors (OF) are required for accurate dose calculation in radiation therapy (Miyashita et al., 2013). In order to calculate the dose accurately, collimator scattering factor $\left(\mathrm{S}_{\mathrm{c}}\right)$ or output factor in air $\left(\mathrm{OF}_{\mathrm{air}}\right)$ for all square and irregular fields with any dimensions should be measured (McKerracher and Thwaites, 2007; Miyashita et al., 2009; Khan and Gibbons, 2014). The output factor (OF) expresses the amount of scattering radiation variations from collimators in the treatment volume and is defined as the ratio of absorbed dose of arbitrary field to that of reference field $\left(10 \times 10 \mathrm{~cm}^{2}\right)($ McKerracher and Thwaites, 2007). $\mathrm{OF}_{\text {air }}$ is measured in the air. Hence, it can be assumed to be equal with the Sc.

The detector system for measuring the Sc, is composed of the ion chamber and appropriate build-up cap that make electronic equilibrium. The build-up cap must be entirely covered by the incident beam for all field sizes to reflect the relative changes of incident fluence (Vadash and Bjärngard, 1993; Miyashita et al., 2009; Iftikhar, 2012; Khan and Gibbons, 2014). To prevent electron contamination for high-energy photon beams, the measuring depth must be sufficiently far away from dmax (5 or $10 \mathrm{~cm}$ ) (Van Gasteren et al., 1991). A mini phantom is used for this purpose.

Output factor in air ( $\left.\mathrm{S}_{c}\right)$ depends on energy and field size (collimator opening) (Huang et al., 1987). For squared fields a small increase in field width and length, will have a small effect on output factor in air. But for rectangular and extremely elongated field shapes, the dependence of $\mathrm{OF}_{\text {air }}$, on field size variations will be more important (Huang et al., 1987). At first it seems that, the $\mathrm{OF}_{\text {air }}$ for a rectangular $\mathrm{a} \times \mathrm{b}$ field must be equal to $\mathrm{b} \times \mathrm{a}$ one. But, as the positions of the jaws in order to provide the rectangular

${ }^{1}$ Department of Radiotherapy and Oncology, Golestan Hospital, ${ }^{2}$ Department of Medical Physics, Medical School, ${ }^{3}$ Department of Radiology Technology, Ahvaz Jundishapur University of Medical Sciences, Ahvaz, Iran. *For Correspondence: raziye.fayazi@gmail.com 
$\mathrm{a} \times \mathrm{b}$ field is different from $\mathrm{b} \times \mathrm{a}$ field, collimator scatter and hence $\mathrm{OF}_{\text {air }}$ will be different for these field sizes (Huang et al., 1987; Vadash and Bjärngard, 1993). The influence of the different jaws opening that make a treatment field, on OFAIR is called collimator exchange effect, which is related to the construction features of linear accelerator head (Zhu et al., 2009).

Since many treatment fields are irregular and $\mathrm{OF}_{\text {air }}$ is often measured as a function of the square field size at the isocenter, the equivalent square fields are used in clinic (Kim et al., 1997; Miyashita et al., 2013). An equivalent square field size is originally that which gives the same central-axis depth dose characteristics as that of an irregular or blocked field (Norvill and White, 2008; Thomas et al., 2014).

One formula for calculating equivalent square field is the 4A/P method (Sterling et al., 1964). But, the 4A/P method does not consider the collimator exchange effect. So, this approach does not have enough precision (Zhu and Ahnesjo, 2004). Furtheremore, the inaccuracy of this formula is greater for large fields (Day and Aird, 1983; Yu et al., 1995). So, Vadash and Bjärngard presented formula $\mathrm{Eq}=\frac{(A+1) X Y}{A X+Y}$ for equivalent squares, which takes into account collimator exchange effect. In the presented equation, ' $A$ ' is a constant specific for each machine's beams. It can be determined experimentally from head scatter factor measurements, or approximated using machine design. For the square fields, $\mathrm{A}$ is equal to 1 (Vadash and Bjärngard, 1993; Kim et al., 1997).

Kim et al., (1998) presented a geometrical weighting factor for calculating A, based on the design of the head linear accelerator.

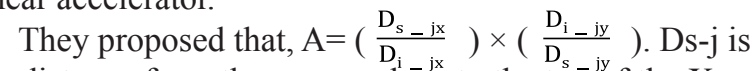
the distance from the source plane to the top of the $\mathrm{X}$ or $\mathrm{Y}$ jaw, and Di-j is the distance from the isocentre plane to the jaw.

Yu et al., (1995) presented the formula $A=\left(\frac{D_{\text {low }}}{D_{u p}}\right)^{2}$, that $\mathrm{D}_{\text {low }}$ and $\mathrm{D}_{\text {up }}$ are distances from the source plane to the down and up jaws, respectively.

Accurate calculation of equal square field size is so important in radiation therapy. Hence, A factor, in the Vadash equation must be determine precisely by considering collimator exchange effect. Therefore, the purpose of this study was to determine the best A factor, for calculation of equal square field size in treatment planning. In the present study, the correction factor (A) was calculated by introducing a new method based on determining output factor in air for different square and rectangular fields for 6 and $18 \mathrm{MV}$ photon beams of linear accelerator.

\section{Materials and Methods}

\section{Analytical method}

Output factor is defined as the ratio of the measured dose $\left(\mathrm{M}_{\mathrm{X} \times \mathrm{Y}}\right)$ in arbitrary field in air on the central axis to reference field $\left(10 \times 10 \mathrm{~cm}^{2}\right)$ in same point (usually the isocenter):

$$
O F_{X \times Y}=\frac{M_{X \times Y}}{M_{10 \times 10}}
$$

Where, $\mathrm{X}$ and $\mathrm{Y}$ represent the outer (lower) and inner (upper) collimator jaws, respectively. For each field, the measured dose consists of the primary and scattered dose portions. The primary dose which comes directly from the source without interactions with matter is independent of collimator opening amount. However, the scatter radiation that generated by the interaction of primary radiation with several head components depends on collimator exchange effect. Therefore, the measured dose for an arbitrary field in air on the central axis $\left(\mathrm{M}_{\mathrm{X} \times \mathrm{Y}}\right)$ can be express as follows:

$$
\mathrm{M}_{\mathrm{X} \times \mathrm{Y}}=\mathrm{P}_{\mathrm{X} \times \mathrm{Y}}+\mathrm{S}_{\mathrm{X} \times \mathrm{Y}}
$$

Which, $\mathrm{P}_{\mathrm{X} \times \mathrm{Y}}$ and $\mathrm{S}_{\mathrm{X} \times \mathrm{Y}}$ represent the primary and scattered doses, respectively. To display the difference between upper and lower jaws on scatter dose (collimator exchange effect), the following notation is applied:

$$
\begin{aligned}
& \mathrm{S}_{\mathrm{a} \times \mathrm{b}}=\mathrm{S}_{\mathrm{a}}{ }^{1}+\mathrm{S}_{\mathrm{b}}{ }^{\mathrm{u}} \\
& \mathrm{S}_{\mathrm{b} \times \mathrm{a}}=\mathrm{S}_{\mathrm{b}}{ }^{1}+\mathrm{S}_{\mathrm{a}}{ }^{\mathrm{u}}
\end{aligned}
$$

The symbols $u$ and 1 refer to the upper $(\mathrm{Y})$ and lower (X) jaws, respectively. "a" and "b" indices represent field dimensions, which determined with collimator opening amount in each cases. Therefore, with respect to Eq. (1), (2), (3) and (4), equations (5) and (6) achieved as follows:

$$
O F_{a \times b}=\frac{M_{a \times b}}{M_{10 \times 10}}=\frac{P_{a \times b}+S_{a \times b}}{M_{10 \times 10}}=\frac{P_{a \times b}+S_{a}^{l}+S_{b}^{u}}{M_{10 \times 10}}
$$

And also,

$$
O F_{b \times a}=\frac{M_{b \times a}}{M_{10 \times 10}}=\frac{P_{b \times a}+S_{b \times a}}{M_{10 \times 10}}=\frac{P_{b \times a}+S_{b}^{l}+S_{a}^{u}}{M_{10 \times 10}}
$$
(6):

Equation (7) was obtained by combining Eq. (5) and

$$
O F_{a \times b}+O F_{b \times a}=\frac{P_{a \times b}+S_{a}^{l}+S_{b}^{u}+P_{b \times a}+S_{b}^{l}+S_{a}^{u}}{M_{10 \times 10}}
$$

Since, the primary dose is independent of the collimator jaws displacement, it can be deduced:

$$
\mathrm{P}_{\mathrm{a} \times \mathrm{b}}+\mathrm{P}_{\mathrm{b} \times \mathrm{a}}=\mathrm{P}_{\mathrm{a} \times \mathrm{a}}+\mathrm{P}_{\mathrm{b} \times \mathrm{b}}
$$

$\mathrm{P}_{\mathrm{a} \times \mathrm{a}}$ and $\mathrm{P}_{\mathrm{b} \times \mathrm{b}}$, show the primary doses of square fields with $\mathrm{a} \times \mathrm{a}$ and $\mathrm{b} \times \mathrm{b}$ dimensions.

Respect to Eq. (8), the Eq. (7) was rewritten as follows:

$O F_{a \times b}+O F_{b \times a}=\frac{P_{a \times a}+S_{a}^{l}+S_{a}^{u}+P_{b \times b}+S_{b}^{l}+S_{b}^{u}}{M_{10 \times 10}}$

And as a result:

$$
\mathrm{OF}_{\mathrm{a} \times \mathrm{b}}+\mathrm{OF}_{\mathrm{b} \times \mathrm{a}}=\mathrm{OF}_{\mathrm{a} \times \mathrm{a}}+\mathrm{OF}_{\mathrm{b} \times \mathrm{b}}
$$

In practical situation, there will be a slight difference $(\Delta)$ between two hand sides of Eq. (10), this difference $(\Delta)$, determined as:

$$
\Delta=\left|\mathrm{OF}_{\mathrm{a} \times \mathrm{b}}+\mathrm{OF}_{\mathrm{b} \times \mathrm{a}}-\mathrm{OF}_{\mathrm{a} \times \mathrm{a}}-\mathrm{OF}_{\mathrm{b} \times \mathrm{b}}\right| \neq 0
$$




\section{Measurement}

Measurement was performed with a Farmer chamber with $0.6 \mathrm{cc}$ volumes in source-surface distance (SSD) $100 \mathrm{~cm}$ and build up cap of Plexiglas, 1.5 and $3.5 \mathrm{~cm}$ equals water, for 6 and $18 \mathrm{MV}$, respectively on a Perimus Plus linear accelerator. Output factors in air were measured for square field sizes $(5 \times 5,6 \times 6,7 \times 7,8 \times 8,9 \times 9,10 \times 10$, $12 \times 12,15 \times 15,20 \times 20,25 \times 25,30 \times 30,35 \times 35,40 \times 40 \mathrm{~cm}^{2}$ ) three times and plotted as a function of the square field size. The relation between output factor and equivalent field size was obtained using MATLAB software (version R2014a) curve fitting toolbox (fitted relation).

In the next step, the output factors were measured for 16 rectangular fields) $5 \times 10,5 \times 20,5 \times 30,5 \times 40,10 \times 20$, $10 \times 30,10 \times 40,20 \times 30 \mathrm{~cm}^{2}$ and conversely).

Six fields for $6 \mathrm{MV}$ and eight fields for 18 were selected. The criteria for selecting these fields, was $\Delta$, smaller than 0.002 for $6 \mathrm{MV}$ and 0.01 for $18 \mathrm{MV}$ energies. After determining the equivalent square field sizes for the selected rectangular fields, using the fitted relation, the Vadash coefficients (A) were calculated for the selected fields by means of Vadash formula for both energies 6, $18 \mathrm{MV}$.

For the each calculated -A-, the equivalent square field size for all rectangular fields was determined by the Vadash formula. The difference between calculated and the measured equivalent square field size which obtained from the fitted relation was determined. The standard deviations $(\sigma)$ were calculated for the mentioned differences and the final coefficient -A-for the each energy was selected with respect to minimum standard deviation ( $\sigma \mathrm{min})$ of the differences.

\section{Results}

The measured output factors for 6 and 18MV energies versus square field size are shown in Figure1.

Equation 12 illustrates the fitted power models with

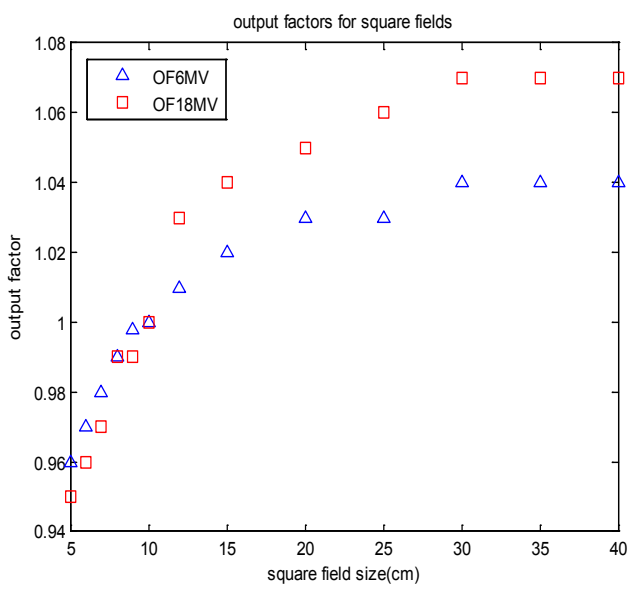

Figure 1. Measured Output Factor for Square Fields in 6 and $18 \mathrm{MV}$

constant value to explain the output factor (OF) as a function of square field size $\left(\mathrm{S}_{\text {equi. }}\right)$ in $\mathrm{cm}$, with $\mathrm{R} 2>0.98$.

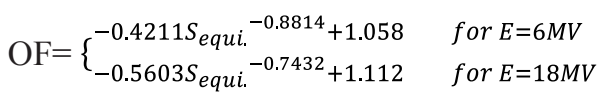

The measured $\mathrm{OF}_{\text {air }}$ and fitted relations (Eq. 12) are shown in figure 2 as functions of square field size.

The measured output factors for square and rectangular fields with values of $\Delta$ in Eq. 11 are shown in Table1.

The minimum value of $\Delta$ was the selecting criteria to choose the values of the Vadash constant coefficient " $A$ ". Therefore, the equivalent square field sizes were calculated for rectangular fields with the lowest $\Delta$ (bold data in Table1) according to Eq.12.

Values of calculated "A", with replacing calculated equivalent square field sizes in Vadash relation, are shown in Table 2. Also, the standard deviations $(\sigma)$ of differences between calculated and the measured equivalent square field sizes are illustrated in Table 2.

The final selected coefficient "A" with respect to

Table1. The Values of $\Delta$ in Eq.11 for Square and Rectangular Fields in 6 and 18MV Energies

\begin{tabular}{|c|c|c|c|c|c|c|c|c|c|}
\hline $\mathrm{E}(\mathrm{MeV})$ & $a \times b$ & $\mathrm{OF}$ & $\mathrm{b} \times \mathrm{a}$ & $\mathrm{OF}$ & $a \times a$ & $\mathrm{OF}$ & $\mathrm{b} \times \mathrm{b}$ & $\mathrm{OF}$ & $\Delta$ \\
\hline 6 & \multirow{2}{*}{$40 \times 5$} & 0.995 & \multirow{2}{*}{$5 \times 40$} & 0.98 & \multirow{2}{*}{$5 \times 5$} & 0.957 & \multirow{2}{*}{$40 \times 40$} & 1.04 & 0.025 \\
\hline 18 & & 0.99 & & 0.75 & & 0.95 & & 1.07 & 0.285 \\
\hline 6 & \multirow{2}{*}{$40 \times 10$} & 1.023 & \multirow{2}{*}{$10 \times 40$} & 1.009 & \multirow{2}{*}{$10 \times 10$} & 1 & \multirow{2}{*}{$40 \times 40$} & 1.04 & 0.008 \\
\hline 18 & & 1.035 & & 1.025 & & 1 & & 1.07 & 0.01 \\
\hline 6 & \multirow{2}{*}{$30 \times 5$} & 1.038 & \multirow{2}{*}{$5 \times 30$} & 1.033 & \multirow{2}{*}{$5 \times 5$} & 0.957 & \multirow{2}{*}{$30 \times 30$} & 1.037 & 0.024 \\
\hline 18 & & 0.996 & & 0.748 & & 0.95 & & 1.068 & 0.28 \\
\hline 6 & \multirow{2}{*}{$30 \times 10$} & 1.022 & \multirow{2}{*}{$10 \times 30$} & 1.013 & \multirow{2}{*}{$10 \times 10$} & 1 & \multirow{2}{*}{$30 \times 30$} & 1.037 & 0.002 \\
\hline 18 & & 1.035 & & 1.026 & & 1 & & 1.068 & 0.006 \\
\hline 6 & \multirow{2}{*}{$30 \times 20$} & 1.031 & \multirow{2}{*}{$20 \times 30$} & 1.031 & \multirow{2}{*}{$20 \times 20$} & 1.029 & \multirow{2}{*}{$30 \times 30$} & 1.037 & 0.004 \\
\hline 18 & & 1.057 & & 1.05 & & 1.055 & & 1.068 & 0.015 \\
\hline 6 & \multirow{2}{*}{$20 \times 5$} & 0.993 & \multirow{2}{*}{$5 \times 20$} & 0.98 & \multirow{2}{*}{$5 \times 5$} & 0.957 & \multirow{2}{*}{$20 \times 20$} & 1.029 & 0.016 \\
\hline 18 & & 0.989 & & 0.746 & & 0.95 & & 1.055 & 0.269 \\
\hline 6 & \multirow{2}{*}{$20 \times 10$} & 1.02 & \multirow{2}{*}{$10 \times 20$} & 1.011 & \multirow{2}{*}{$10 \times 10$} & 1 & \multirow{2}{*}{$20 \times 20$} & 1.029 & 0.002 \\
\hline 18 & & 1.031 & & 1.026 & & 1 & & 1.055 & 0.002 \\
\hline 6 & \multirow{2}{*}{$10 \times 5$} & 0.983 & \multirow{2}{*}{$5 \times 10$} & 0.976 & \multirow{2}{*}{$5 \times 5$} & 0.957 & \multirow{2}{*}{$10 \times 10$} & 1 & 0.002 \\
\hline 18 & & 0.983 & & 0.731 & & 0.95 & & 1 & 0.238 \\
\hline
\end{tabular}


Table 2. Calculated "A" for the Fields with the Lowest $\Delta$

\begin{tabular}{|c|c|c|c|c|c|c|c|}
\hline $6 \mathrm{MV}$ & & & & $18 \mathrm{MV}$ & & & \\
\hline $\begin{array}{l}\text { Field } \\
\text { sizes }\end{array}$ & $\Delta$ & A & $\sigma$ & $\begin{array}{l}\text { Field } \\
\text { sizes }\end{array}$ & $\Delta$ & A & $\Sigma$ \\
\hline $10 \times 5$ & 0.002 & 1.42 & 0.98 & $40 \times 10$ & 0.01 & 0.72 & 3.42 \\
\hline $5 \times 10$ & 0.002 & 1.29 & 1.07 & $10 \times 40$ & 0.01 & 3.13 & 2.75 \\
\hline $20 \times 10$ & 0.002 & 2.25 & 1.71 & $20 \times 10$ & 0.002 & 1.06 & 2.44 \\
\hline $10 \times 20$ & 0.002 & 1.97 & 1.37 & $10 \times 20$ & 0.002 & 1.55 & 1.9 \\
\hline $30 \times 10$ & 0.002 & 1.37 & 1.01 & $30 \times 10$ & 0.006 & 0.86 & 2.93 \\
\hline $10 \times 30$ & 0.002 & 2.2 & 1.65 & $10 \times 30$ & 0.006 & 2.32 & 2.12 \\
\hline $20 \times 30$ & 0.004 & 1.96 & 1.36 & - & - & - & - \\
\hline $30 \times 20$ & 0.004 & 0.51 & 3.6 & - & - & - & - \\
\hline
\end{tabular}

minimum standard deviation ( $\sigma \mathrm{min})$ of the differences for 6 and $18 \mathrm{MV}$ energies are bolded in Table 2.

\section{Discussion}

Photon beam data, such as percentage depth doses, dose profiles, and output factors (OF) are required for dose calculation in radiation therapy. The $\mathrm{OF}_{\text {air }}$ can be determined by energy fluence of primary and scattered photons and backscattered radiation from collimator jaws. Scattered photons generated from primary collimator and flattening filter was account for $90.0 \%$ of total scattered photon fluence. Scattered photons from flattening filter decreased slowly as distance from central axis increases (Miyashita et al., 2013). By increasing the field size, the collimator opening increases and the greater surface is irradiated. The Y collimator also have a greater effect on the $\mathrm{OF}_{\text {air }}$ which is called collimator exchange effect (Vadash and Bjärngard, 1993).

Collimator exchange effect should be taken into consideration, due to its impact on dose calculation via OF (Jensen, 2003). Besides, "A" value in Vadash formula minimizes the collimator exchange effect, in calculating the equivalent squares which plays an important role in the patient dose calculation and treatment planning. The value of "A" constant depends on head accelerator design and should be calculated for each machine and energy.

In this study, a new simple protocol based on the selection of best dosimetric data was proposed to obtain "A" value in Vadash relation to predict the equivalent square field size for rectangular fields which compensates collimator exchange effect in $\mathrm{OF}_{\text {air. }}$. Table 1 data shows that the $\mathrm{OF}_{\text {air }}$ has small variations with field size. For the linear accelerator used in this study the values range from about 0.983 to 1.038 in $6 \mathrm{MV}$ and from 0.731 to 1.05 in $18 \mathrm{MV}$, for the measured fields.

Collimator exchange effect is obviously seen in Table 1 data. For example, the $\mathrm{OF}_{\text {air }}$ for a $30 \times 5 \mathrm{~cm}^{2}$ field in 18 $\mathrm{MV}$ is greater than $\mathrm{OF}_{\text {air }}$ of $5 \times 30 \mathrm{~cm}^{2}$ field due to using the upper jaw to build the field length which has the greater effect on scatter. The minimum $\Delta \mathrm{min}$ was equal to 0.002 for $10 \times 30,10 \times 20$ and $5 \times 10 \mathrm{~cm}^{2}$ and the maximum difference was 0.025 for $5 \times 40 \mathrm{~cm}^{2}$ for $6 \mathrm{MV}$ energy. For $18 \mathrm{MV}$ energy the minimum $\Delta \min$ was equal to 0.002 for $10 \times 20 \mathrm{~cm}^{2}$ and the maximum difference was 0.285 for $5 \times 40 \mathrm{~cm}^{2}$.

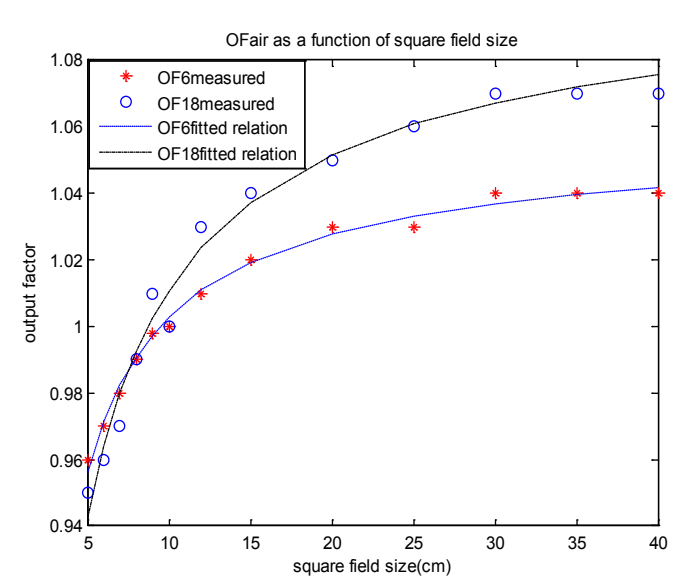

Figure 2. The Measured $\mathrm{OF}_{\text {air }}$ and Fitted Relations (Eq. 12) are Shown in Figure 2 as Functions of Square Field Size.

As shown in Table 2, the values of " $\mathrm{A}$ " $=1.42$ and 1.55 with respect to $\sigma \mathrm{min}=0.98$ and 2.3 for 6 and $18 \mathrm{MV}$ energies were selected, respectively among 6 calculated values for "A" constant. Vadash et al (Vadash and Bjärngard, 1993) obtained value of 1.8 for " $A$ " constant for Philips accelerator in 6 and 25MV. M.K.Yu et al (Yu et al., 1995) divided measurements in two groups. In the first group field sizes were defined by $\mathrm{X}$ and $\mathrm{Y}$ jaws. Field sizes were determined by MLC and Y jaws where $\mathrm{X}$ jaw settings were fixed in constant distance from the centerline for the second group. They reported constant value 1.7 for Varian Clinac 600CD and 1.78 and 1.85 for Varian Clinac $2300 \mathrm{CD}, \mathrm{X}$ and $\mathrm{Y}$ jaws in 6 and 15MV respectively and 3.38 for Varian Clinac 2300CD, MLC and Y jaws in 6MV. Kim et al (Kim et al., 1998) represented a function for " $\mathrm{A}$ " value which depended on distances of source to every jaws and detector plane. The obtained value for Varian Clinac 2300CD in 8MV was 1.5. Norvill et al (Norvill and White, 2008) calculated $\mathrm{OF}_{\text {air }}$ for square and rectangular fields in 6 and $18 \mathrm{MV}$ and equivalent square values for the rectangular field sizes by fitting interpolation and substituting values of -A- range (1-2.6) in Vadash formula and finally represented A value 1.8 for Siemens Oncor linear accelerator.

In conclusion, a new simple protocol based on dosimetry data was proposed to calculate " $\mathrm{A}$ " value in Vadash relation to predict the equivalent square field size for rectangular fields which compensates collimator exchange effect in $\mathrm{OF}_{\text {air }}$. The coefficients " $\mathrm{A}$ " $=1.42$ and 1.55 have the minimum standard deviations for differences between calculated and measured equivalent field sizes in 6 and $18 \mathrm{MV}$ energies, respectively. Hence, these values were selected as the final coefficients for equivalent field size assessment in Vadash relation.

The results of proposed protocol presented good agreement with other studies and can be used for calculation of equivalent square field sizes. However, it is necessary to accomplish and complete the study by more dosimetry experiments to expand the results and improve the protocol. 


\section{Acknowledgments}

The authors thank the office of Vice-Chancellor of Ahvaz Jundishapur University of medical sciences for financial support with grant number (U-96056).

\section{References}

Day M, Aird E (1983). The equivalent-field method for dose determinations in rectangular fields. Br J Radiol Suppl, 17, 105.

Huang PH, Chu J, Bjärngard BE (1987). The effect of collimator backscatter radiation on photon output of linear accelerators. Med Phys, 14, 268-9.

Iftikhar A (2012). Measurements of output factors using different ionization chambers and build up caps. Iran J Radiat Res, 10, $95-8$

Jensen J (2003). Output-factors for squared, rectangular, and elongated photon fields of medical linear accelerators. Z Med Phys, 14, 113-7.

Khan FM, Gibbons JP (2014). treatment planning I: isodose distributions. In 'Khan's the physics of radiation therapy', Eds Lippincott Williams and Wilkins, 170-95.

Kim S, Palta JR, Zhu TC (1998). A generalized solution for the calculation of in-air output factors in irregular fields. Med Phys, 25, 1692-701.

Kim S, Zhu TC, Palta JR (1997). An equivalent square field formula for determining head scatter factors of rectangular fields. Medi Phys, 24, 1770-4.

McKerracher C, Thwaites DI (2007). Head scatter factors for small MV photon fields. Part I: A comparison of phantom types and methodologies. Radiother Oncol, 85, 277-85.

Miyashita H, Hatanaka S, Fujita Y, et al (2013). Quantitative analysis of in-air output ratio. $J$ Radiat Res, 54, 553-60.

Miyashita H, Hatanaka S, Fujita Y, et al (2009). Head scatter factor calculation considering characteristics of scattered photon. World congress on medical physics and biomedical engineering, September 7-12, 2009, Munich, Germany, 2009. Springer, pp 700-3.

Norvill C, White P (2008). An investigation of equivalent square formulas. Australas Phys Eng Sci Med, 31, 151-3.

Shalek RJ (1977). Determination of absorbed dose in a patient irradiated by Beams of $\mathrm{X}$ or Gamma rays in radiotherapy procedures. Med Phys, 4, 461.

Sterling T, Katz L, Perry H (1964). Derivation of a mathematical expression for the per cent depth dose surface of Cobalt 60 Beams and visualisation of multiple field dose distributions. Br J Radiol, 37, 544-50.

Thomas S, Eaton D, Tudor G, et al (2014). Equivalent squares for small field dosimetry. Br J Radiol, 81, 897-901.

Vadash P, Bjärngard B (1993). An equivalent-square formula for head-scatter factors. Med Phys, 20, 733-4.

Van Gasteren J, Heukelom S, Van Kleffens H, et al (1991). The determination of phantom and collimator scatter components of the output of megavoltage photon beams: measurement of the collimator scatter part with a beam-coaxial narrow cylindrical phantom. Radiother Oncol, 20, 250-7.

Van Herk M (2004). Errors and margins in radiotherapy. Seminars in radiation oncology. Elsevier, pp 52-64.

Yu M, Murray B, Sloboda R (1995). Parametrization of head-scatter factors for rectangular photon fields using an equivalent square formalism. Med Phys, 22, 1329-32.

Zhu T, Ahnesjo A (2004). Interpretation of in-air output ratio and its impact on dose calculation. AAPM refresher course notes.pp, 35.

Zhu TC, Ahnesjö A, Lam KL, et al (2009). Report of AAPM therapy physics committee task group 74: In-air output ratio,
Calculation of Equivalent Square Fields in Radiation Therapy

Sc, for megavoltage photon beams. Med Phys, 36, 5261-91.

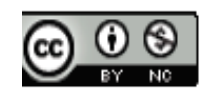

This work is licensed under a Creative Commons AttributionNon Commercial 4.0 International License. 